\title{
Design manufacturing integration and flight testing of a health monitoring system for a prototype unmanned airborne vehicle
}

\author{
Craig P Lawson and Guido AI Monterzino \\ School of Engineering, Cranfield University
}

\begin{abstract}
This paper describes the design, development, build and flight testing of a health monitoring system for the landing gear and the electrical power system on board the Demon prototype Unmanned Airborne Vehicle. Demon is a flying technology demonstrator which successfully flew in September 2010. The Demon can achieve pitch and roll control without the use of hinged control surfaces, by instead using fluidic devices based on the Coanda effect, attaining low-maintenance, highmanoeuvrability operations. A vehicle health monitoring system was added on board between the first and the second flight test campaigns. The integration of the health monitoring system into the vehicle is discussed as a whole. The key health monitoring sub-systems include data logging and real-time measurement of several parameters. This includes systems to measure Voltage and current from the main batteries, landing gear stress, suspension travel, wheel hub acceleration and shock absorber pressure. Wherever possible, the use of commercially available components was maximised to minimise development time and cost. Some example results of system health monitoring during flight trials are presented.
\end{abstract}

\section{Keywords}

Aerospace; unmanned airborne vehicle; health monitoring system

\section{Acronyms}

CBM Condition based maintenance

COTS Commercial off the shelf 
DAQ Data acquisition

EPSRC Engineering and physical sciences research council

FLAVIIR Flapless airborne vehicle integrated industrial research

IVHM Integrated vehicle health monitoring

LVDT Linear voltage displacement transducer

MEM Micro electro-mechanical machine

MTOW Maximum take-off mass

RVDT Rotary voltage displacement transducer

UAV Uninhabited airborne vehicle

UCAV Uninhabited combat airborne vehicle

VHM Vehicle health monitoring

\section{Background}

According to [1], Vehicle Health Monitoring (VHM) is the transformation of system data into information to support operational decisions that result in minimised maintenance actions, improved readiness and availability, reduced redundancies, product life extension and improved environmental impact. This is probably the most general concept of health monitoring and it is applicable to any system including an automotive, rail, aircraft, or power generation plant.

According to [2], from a safety perspective, VHM capabilities will enable the rapid detection and diagnosis of adverse events, essential to the safe operation of the vehicle, and will enable the estimation of the condition severity and the remaining useful life within confidence bounds for the affected systems.

Over the past twenty years, health monitoring has vastly extended its capability to monitor systems for operational suitability. Studies on various systems have progressively revealed means of deducing the health of their sub-parts, such as specific aero engine components [3], landing gear [4, 5], airframe structure, and many more. In most cases, researchers mapped the signatures of known areas of concern and determined methods to detect these signatures as they occurred. In safety critical applications, the origin was the development of early detection techniques for termination of operation of the system before severe loss occurred. Amongst the first health monitoring systems are those for spacecraft [6] and for aero-engine in-flight monitoring [7]. Each was designed to identify faults and to notify the team who operates the vehicle of existing and pending maintenance or safety issues as they develop. 
In some new aircraft, such as the latest Boeing passenger aircraft (B787), health monitoring devices allow the vehicle to call ahead to a ground station while en-route to report upcoming necessary maintenance, based on detected issues [8]. With such a capability, the conventional scheduled maintenance methods should then be replaced by a more efficient maintenance method, such as the Condition Based Maintenance (CBM) method. Following this philosophy, the ultimate solution would appear to be an Integrated Vehicle Health Management (IVHM) system, which detects and isolates the fault using distributed diagnostics devices, predicts the life of the component undergoing failure using prognostics tools, and estimates the useful life left which, enables a CBM approach to be adopted. Going forward, the adoption of IVHM is expected to play a major role in reducing the maintenance effort and cost, improving the safety and increasing the profit of the operators by means of increased aircraft availability. In addition to civil transport aircraft, manned military aircraft currently under development are being designed with IVHM as an integrated function [9, 10]. With their inherent level of autonomy, it also makes sense for new unmanned military aircraft to adopt an IVHM approach.

Distinct challenges exist with adopting a VHM approach for existing aircraft. Most legacy aircraft feature airframe systems which do not suit an integrated approach when trying to design a vehicle health monitoring system for instance, as part of a mid-life upgrade. An evident example comes from the fact that the typical legacy avionics are based on federated architectures. Also, typically the onboard electric distribution system is routed through wings and fuselage in sealed looms, which can only be modified or replaced at substantial expense. Nonetheless, the legacy aircraft are expected to be in the service for their originally designed life and beyond. Hence, non-intrusive VHM concepts are to be adopted into legacy aircraft as a retro-fit to support the extended service life effectively.

The work reported in this paper is focused on VHM systems for a prototype UAV which, had already flown without any monitoring system. This requires the clear understanding of the existing airframe systems and the avionics architecture to design the VHM system. So, the objectives of this investigation are:

- Study of the architecture of legacy aircraft systems to be monitored

- Identifying suitable non-intrusive additional sensors

- Identifying a suitable Data Acquisition system (DAQ)

- Study and harmonisation of power requirements of sensors and DAQ

- Functional and physical integration of the health monitoring systems into the aircraft 
- Off-board analysis of available health data.

\section{Prototype vehicle description}

The Demon UAV (see Figure 1) was developed and flight tested during the FLAVIIR project. The FLAVIIR project [11-16] was a five-year research programme looking at technologies for future UAVs and it was funded jointly by BAE Systems and by the Engineering and Physical Sciences Research Council (EPSRC) in the UK. Managed jointly by BAE Systems and Cranfield University, the project included nine additional collaborating university partners. The research programme covers all essential aspects of aeronautical technology integration for the next generation of advanced UAV and UCAV (Unmanned Combat Air Vehicles) concepts. The focus for the research was the 'Grand Challenge' proposed by BAE Systems: “To develop technologies for maintenance-free, low-cost UAV without conventional control surfaces and without performance penalties over conventional craft". The principal goal of this ambitious programme of research was to design, build and fly a small, but representative, UAV embodying the integrated technologies developed in the various research studies comprising the project.

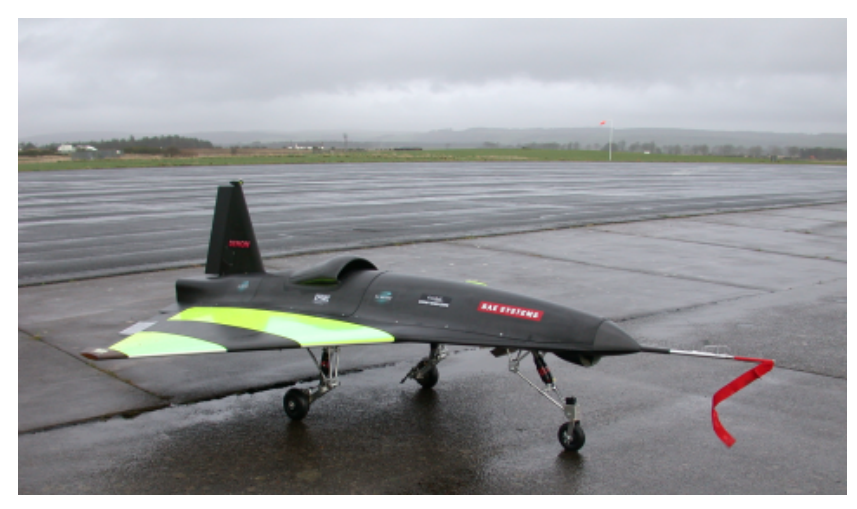

Figure 1. The Demon UAV on the runway.

In particular, it is intended to demonstrate the feasibility of total flight control utilising 'flapless' technologies. In the context of the project, 'flapless' flight control is interpreted to mean circulation control on the wings by means of trailing edge blowing, and thrust vectoring the exhaust from the small propulsive gas turbine engine, as indicated in Figure 2. 


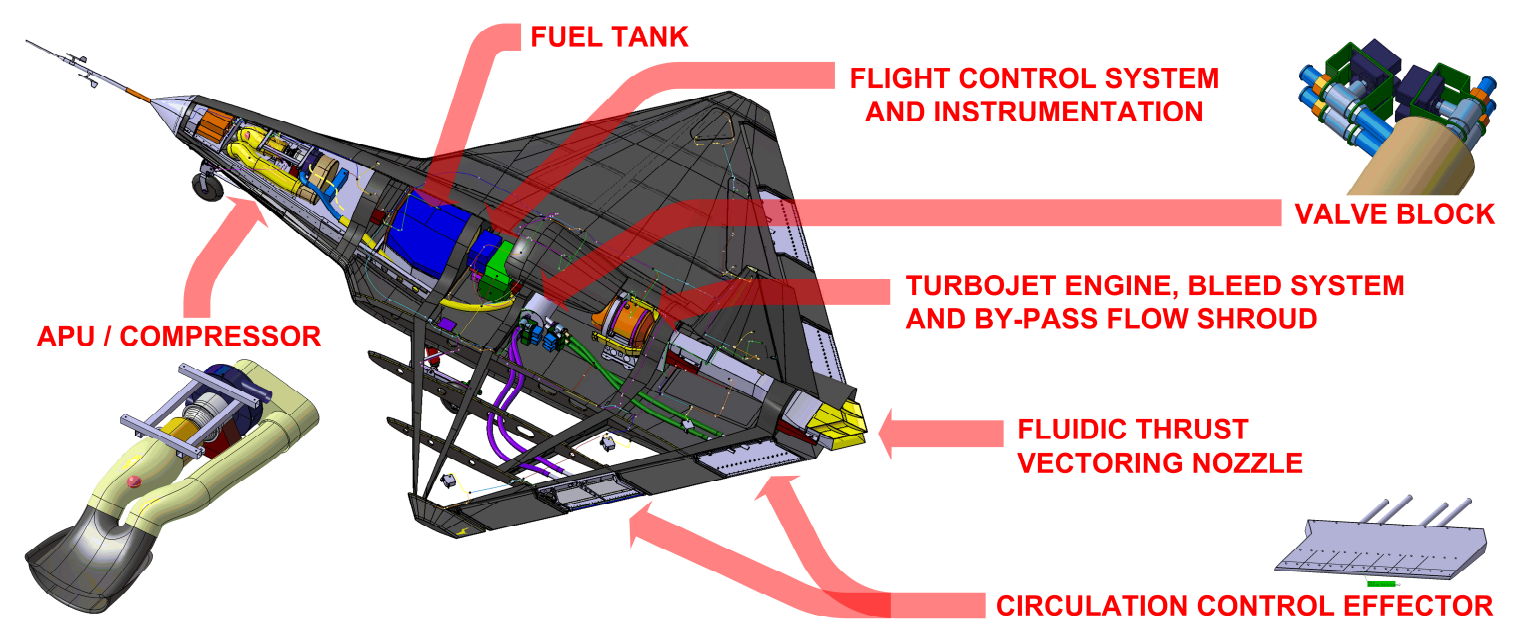

Figure 2. The Demon UAV showing the layout of the major systems.

Demon achieves pitch and roll control without the use of hinged control surfaces, but instead by using fluidic devices and the Coanda effect, powered by a dedicated pneumatic system. The Demon can also be flown in a conventional mode by using hinged control surfaces driven by electromechanical actuators.

The Demon is the largest flying demonstrator within the FLAVIIR project. The Demon has a maximum take-off weight (MTOW) of $90 \mathrm{~kg}$ and a diamond shaped wing plan-form of 2.5 metres in span and 3 metres in length, and $3.13 \mathrm{~m}^{2}$ in area. The vehicle has a stall speed of $29 \mathrm{~m} / \mathrm{s}$ and a maximum operating speed of $65 \mathrm{~m} / \mathrm{s}$ limited by the safety case. The designed dive speed is $90 \mathrm{~m} / \mathrm{s}$. The installed thrust available from the turbojet engine is $360 \mathrm{~N}$.

\section{Retrospective vehicle health monitoring systems}

Generally, vehicles are kept in service beyond their original design life. Most vehicles currently under design and development will include IVHM concepts as an integral part of the design. Conversely, most existing designs cannot be considered 'health-monitoring ready'. Therefore, the typical VHM system for legacy aircraft is to be designed by focusing on the fact that it will have to be retrofitted on a pre-existing fleet of commercial or military aircraft. The legacy aircraft VHM system should be as much as possible a self-contained, independent system which incorporates state-of-the-art sensor 
technology and is easy to integrate with the existing on-board systems, hence minimising modifications. It is also important to investigate using information generated by existing sensors, that may be of use for health monitoring purposes. This can be seen as 'free' data for the retro-fitted VHM system, having no additional impact on the existing aircraft. In light of these principles, it can be clearly seen that implementing VHM technologies on legacy aircraft is a great challenge.

In the case of the Demon UAV, said task turned out to be particularly challenging because of its unusual wing and fuselage volume distribution, resulting from the blended wing body configuration. Also, since the Demon is a one-off prototype, access to systems proved to be more difficult than on a production aircraft due to the vast amount of special, non-standard solutions on-board. Lastly, the limitations posed by the $90 \mathrm{~kg}$ MTOW class made it more challenging to source suitable Commercial Off-The-Shelf (COTS) components to meet mass and performance constraints.

As a result, a trade-off was necessary as to what to monitor. During the down-selection process, the batteries and the landing gear were identified as the most important items to monitor. The reasons are, respectively:

- Electrical Power System: There is no mechanically driven electric power generation on-board the Demon UAV, hence the four 7.4V, 6.3Ah main batteries are the sole source of electric power for flight controls. They are essential for the safe course of the flight. If more than two batteries out of four are out of order, it would be impossible to control the vehicle and not even the fail-safe system built into the radio receivers would operate.

- Landing Gear: The Demon UAV was designed to demonstrate flapless manoeuvrability on a scaled-down, UCAV type of platform. Hence, a high wing loading $\left(282 \mathrm{~N} / \mathrm{m}^{2}\right)$, which implicitly makes the chances of a hard landing more likely, and its consequences harsher.

The aim of the project is to demonstrate that it is possible to design, integrate and flight test health monitoring systems to successfully monitor these aircraft systems. It is an important goal to reach flight testing of the system because; it is not until this stage that all of the integration and operational challenges can be assured to be overcome.

The final monitoring system was therefore, designed to acquire the following real-time data:

1. battery voltage measurement

2. battery current measurement

3. landing gear stress measurement

4. landing gear suspension travel measurement 
5. landing gear acceleration measurement at wheel hubs

6. landing gear shock absorber pressure measurement

The fuselage mounted parts of the health monitoring system are shown at aircraft level in Figure 3, along with some of the major aircraft systems.

Figure 3. Schematic of the Demon UAV health monitoring system - fuselage mounted components.

\section{Demon VHM system architecture description}

The vehicle health monitoring system on board the Demon UAV is divided into two sections: battery data and landing gear data.

The battery data are based on instant voltage and instant current drain recorded from each of the four 7.4V Li-Po batteries that energize the Demon flight control power system. The nominal logging frequency for battery data is $5 \mathrm{~Hz}$. The data acquisition module is made up of two COTS units (DataQ Instruments $^{\mathrm{TM}}$ DI-710), providing 16 analog channels each (see Figure 4). Therefore, eight channels out of thirty-two in total are dedicated to battery data; four voltages and four currents (one per 7.4V battery) are logged simultaneously. No remote voltage transducers were needed as the A/D converter 
of DataQ Instruments ${ }^{\mathrm{TM}}$ DI-710 features a suitable input range of between $\pm 10 \mathrm{~V} \pm 10 \mathrm{mV}$. Hence, it was suitable to provide a direct measurement of the voltage signals from all sensors on board.

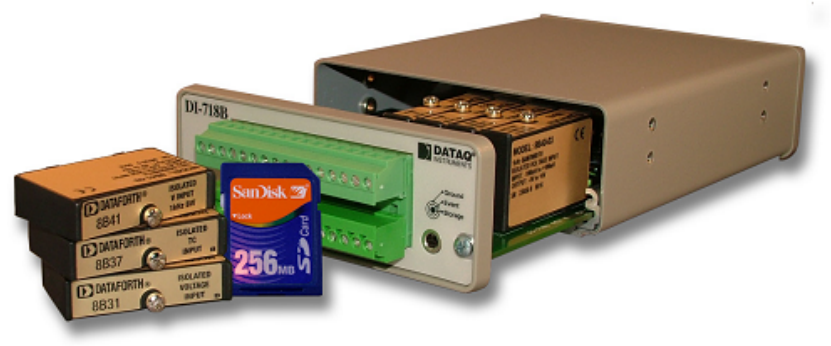

Figure 4. DAQ modules used for Demon VHM: DataQ Instruments ${ }^{\mathrm{TM}}$ DI-710.

The Demon health monitoring system is powered by an independent $18.5 \mathrm{~V} 3.8 \mathrm{Ah}$ Li-Po battery, which energises both the data acquisition module and all the sensors on board via high-frequency, lowripple DC/DC converters (switching voltage regulators). All voltage regulators utilised in the VHM system are from the R-78HB series by Recom ${ }^{\mathrm{TM}}$. Their switching frequency is $400 \mathrm{kHz}$, which in comparison with the fastest channel of the on-board DAQs is greater by a ratio of over 26:1. At said switching frequency, the value of the average ripple on the regulated output is approximately $9 \mathrm{mV}$. As a result of the two factors described above, the voltage fluctuation downstream with respect to the DC/DC converters proved not to be appreciable in the logged data sets. One more reason for the choice of the R-78HB series is that it is capable of delivering a maximum efficiency of $96 \%$, which in turn meant that there was no need for heat sinks, which saved volume and mounting costs.

The other section of the Demon VHM system is related to landing gear data. The Demon's landing gear is shown in Figure 5. Seventeen more channels are utilized for landing gear monitoring as follows:

- 3 channels for bending moment measurements (one per landing gear leg)

- 2 channels for torsion measurements (main landing gear legs only)

- 3 channels for suspension travel measurements (one per landing gear leg)

- 6 channels for acceleration measurements at wheel hubs, along X, Y and Z axes (main landing gear legs only)

- 3 channels for pressure measurements of the shock absorbers (one per landing gear leg). 
Therefore, seven channels were left unused in the data logging system for future growth. The design logging frequency of all of the data listed above is $15 \mathrm{kHz}$, for systems identification purposes. It is also possible to use these data to derive the actual overall weight of the vehicle while on the ground, and its instantaneous center of gravity position. The accuracy of these non-directly measured values is augmented by cross-comparing three redundant sources; bending moment on landing gear trailing links, torsion on the same parts, and pressure inside the shock absorber together with the trailing arm deflection.

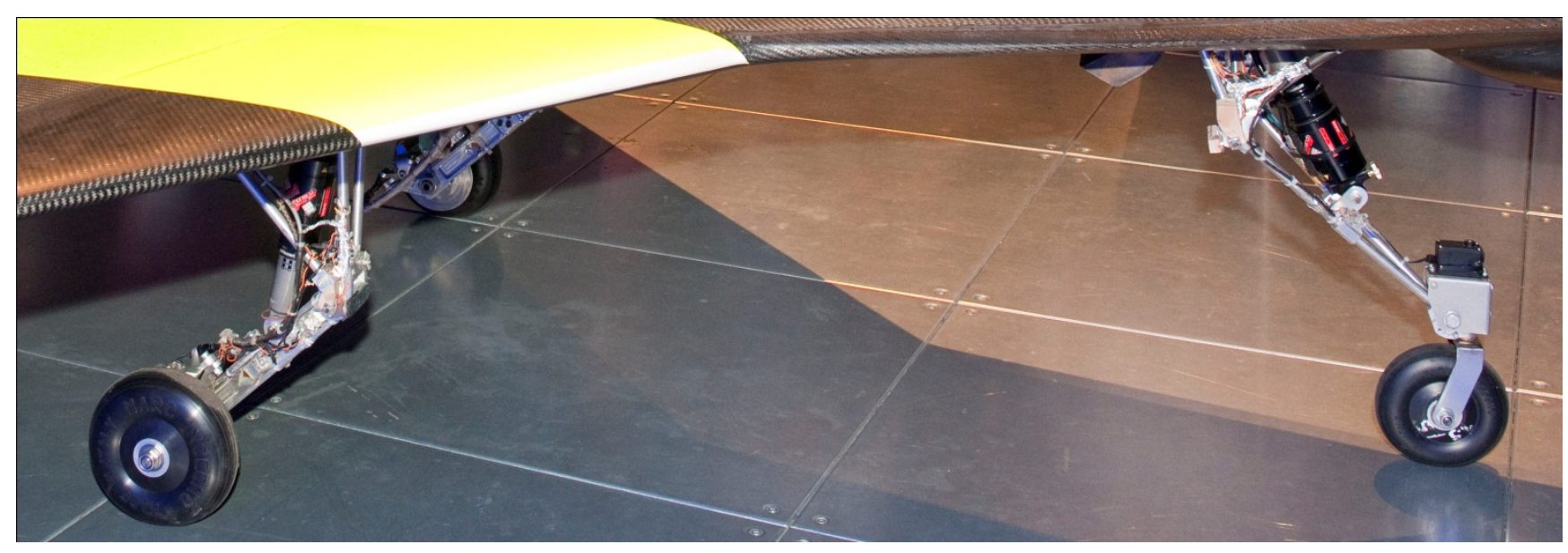

Figure 5. Demon landing gear.

\section{Sensor technology}

A vital step in monitoring the health of any system is to measure the physical quantity of the measurable values at the test points. The test points provide an indication of status which, is compared with the deterministic behaviour of the components. Thus, the assessment of the component health status is facilitated. The following are some of the traditional and novel transducers that have been adopted for the Demon VHM.

\section{Electrical power system current and voltage sensors}

Conventional COTS current clamps were used, mounted on the cables between the batteries and the power distribution module. The power distribution module has an onboard computer which, measures the voltage across each battery. This data was taken and recorded in the VHM DAQ. 
All landing gear stress measurement was based on conventional resistive strain gauges. Given the complexity of the landing gear structure, it has been considered preferable to maximise the predictability of sensor behaviour rather than maximising the electrical efficiency, which could have been achieved by piezoelectric or semiconductor-based strain sensors. The landing gear strain measurement system is illustrated in Figure 6.
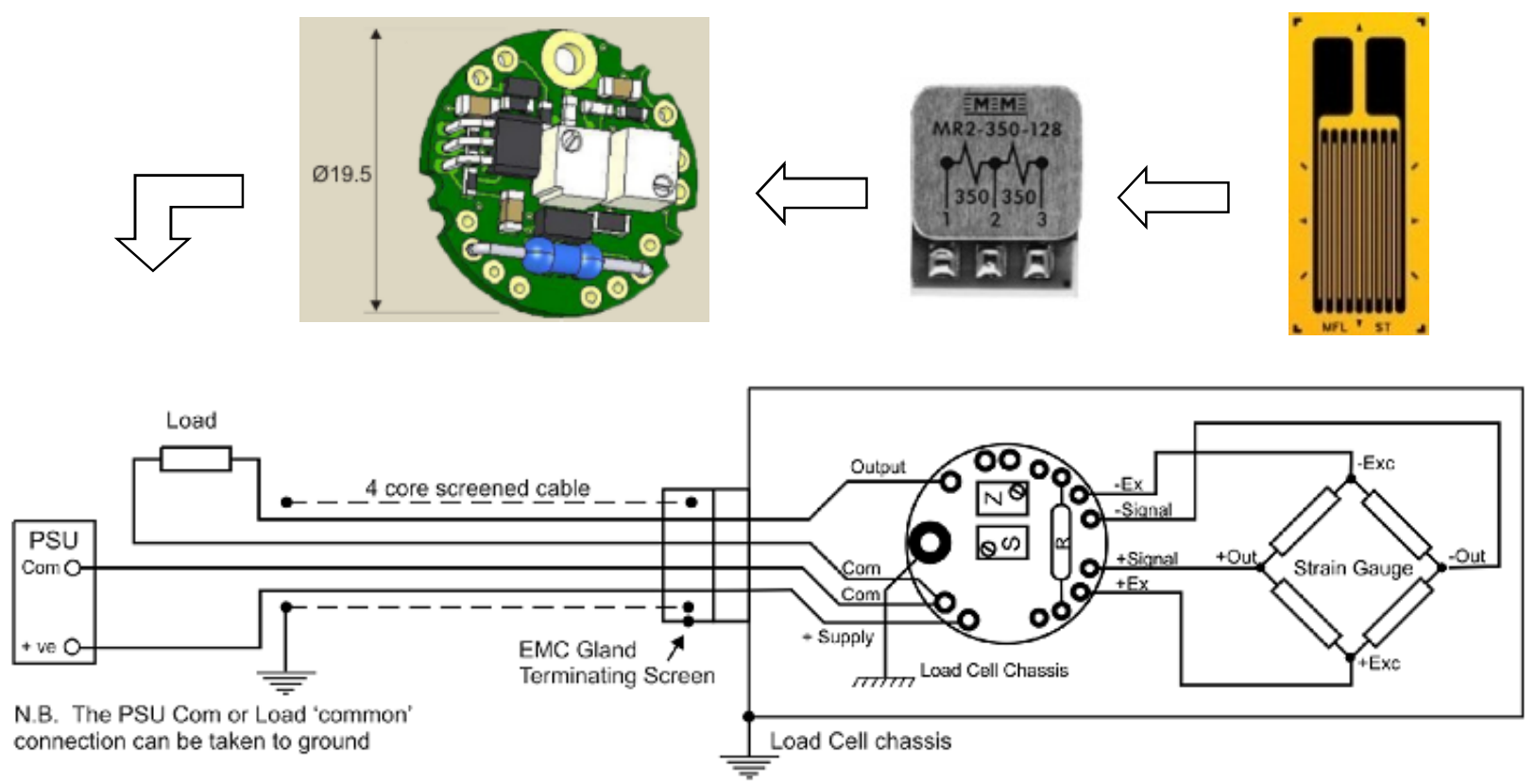

Figure 6. Strain gauge signal conditioning on Demon landing gear structure.

All of the strain gauges utilised on Demon are standard Vishay ${ }^{\mathrm{TM}}$, unidirectional $120 \Omega$ gauges for application on steel or aluminium alloys. They feature $6 \mathrm{~mm}$ of active gauge length. A total of ten strain gauges can be found on the Demon landing gear legs working in pairs in half-Wheatstone-bridge setup. There are two bridges on each main landing gear leg (to sense both bending and torsion moments) and a single bridge on the nose leg (sensing bending moment only). A twin-resistor completion unit, visible in its metal finish in Figure 7, provides an interface for each half-bridge to a sub-miniature conditioning and amplifying analogue module (LCM Systems Ltd ICA1S). 


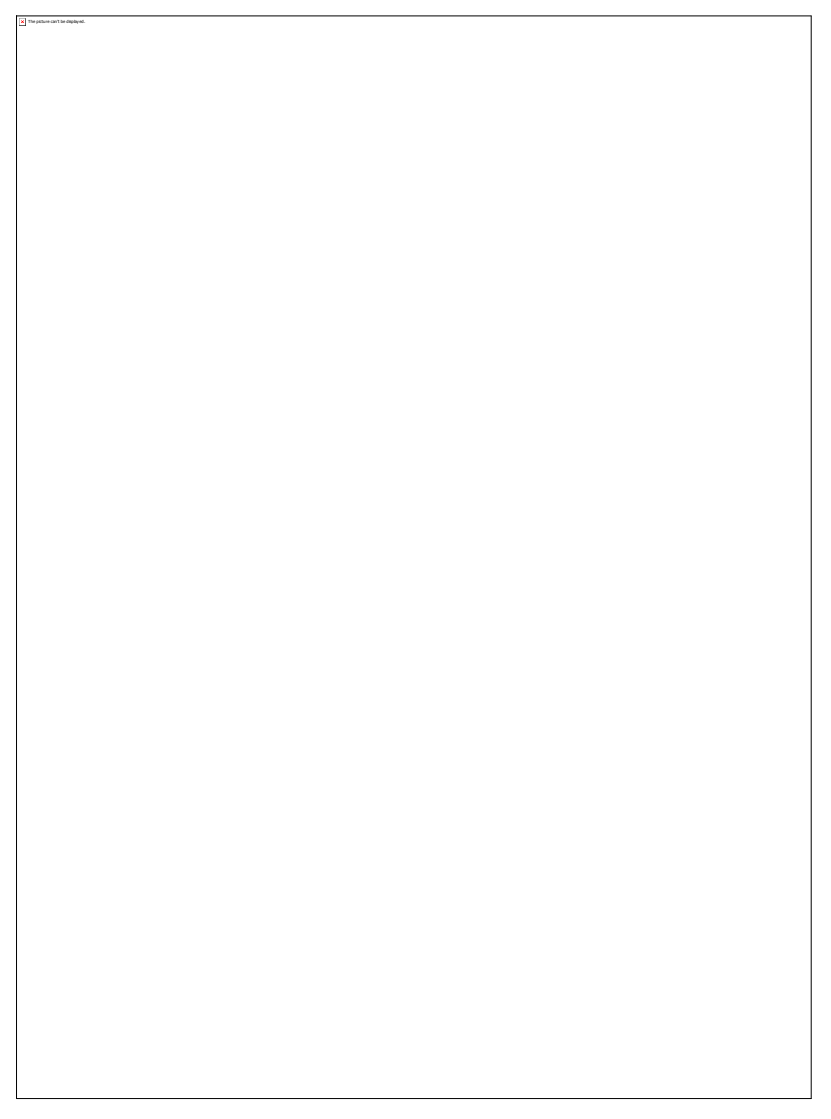

Figure 7. Demon starboard main landing gear leg.

\section{Acceleration sensors}

Similarly to the choice reasoning that was made in the case of the strain sensors, Microelectromechanical (MEM) accelerometers were chosen rather than capacitive type, in order to increase the robustness of the system. In fact, although the sensitivity of a capacitive accelerometer is superior (especially in the low frequency range), and they can be operated in servo mode to achieve higher linearity, the tolerance to over-full-scale excitation is substantially less than for a MEM type. The accelerometer that was selected for this application is the analogue model KXM52-1050 by Kionix ${ }^{\mathrm{TM}}$ (see Figure 8 and Figure 9). It features 3 -axis analogue output ranging between $\pm 5 \mathrm{~g}$, and it produces a signal which is linearly proportional to the exerted acceleration $(0.66 \mathrm{~V} / \mathrm{g}$ with an excitation voltage of 3.3 V). Considering that the installation of the accelerometers on the Demon landing gear is rigidly in contact with the wheel hub, it became evident that values well in excess of the nominal full-scale value of $5 \mathrm{~g}$ are expected over the operational life of the vehicle, also considering the solid tyres (semipneumatic type). Shock load tolerance of the chosen accelerometers is up to $4600 \mathrm{~g}$ in a $0.5 \mathrm{~ms}$ halversine with no damage occurring, regardless of the status of activity (powered or unpowered). 


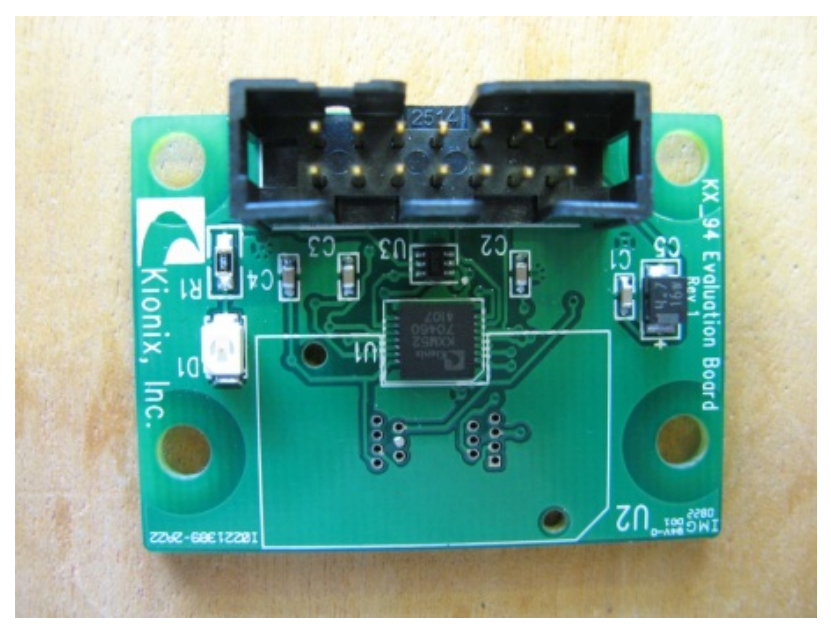

Figure 8. Demon wheel-hub mounted accelerometer KXM52-1050 by Kionix ${ }^{\mathrm{TM}}$.

Moreover, MEM accelerometers offer a lower package weight and a higher temperature range, which suited the application in question as the inboard part of the main wheel hubs is immersed in the Auxiliary Power Unit exhaust flow. As a result of this, the aluminium surface where the accelerometers are located reaches approximately $60{ }^{\circ} \mathrm{C}$ during taxiing. The accelerometers have a working range of -40 to $12560{ }^{\circ} \mathrm{C}$. However, offset increases and sensitivity decreases with temperature. Therefore, the addition of a thermal shielding protection on all sensors placed on the main landing gear legs was implemented. 


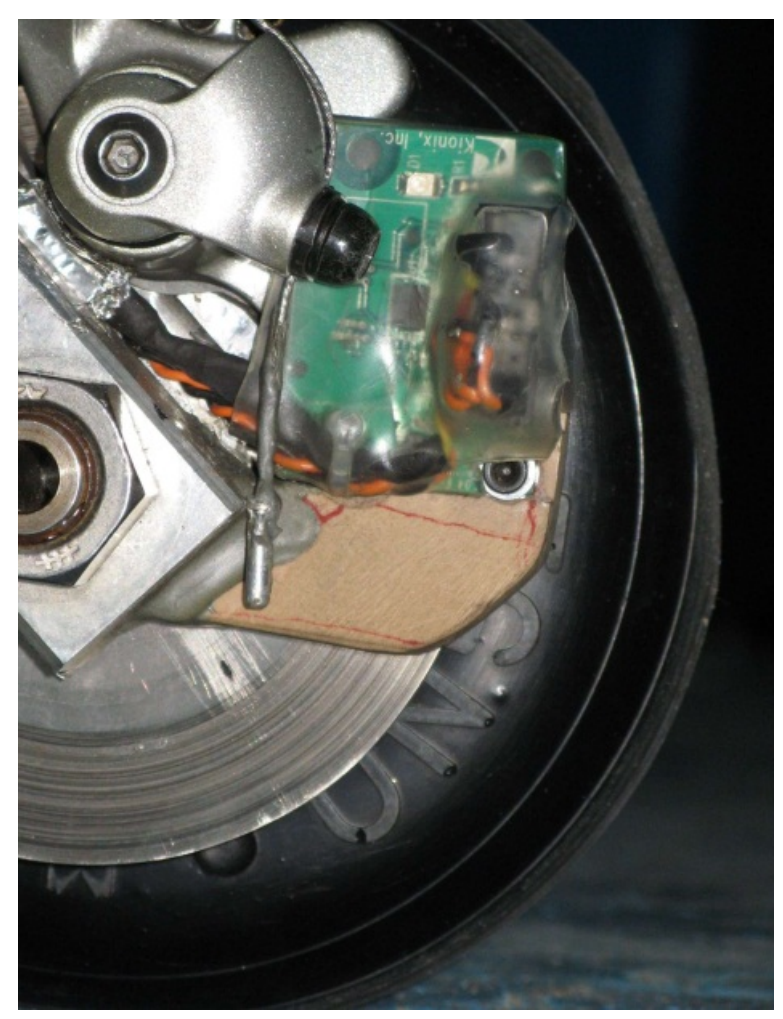

Figure 9. Demon wheel-hub mounted acceleration sensors.

Each of the accelerometers' three axes signals are utilized. $\mathrm{X}$ and $\mathrm{Z}$ are used to capture two components of impact acceleration in the vertical plane, and the resultant vector is calculated based on the measured angle of deflection (see Section 5.4). Y is used to assess lateral movement on trailing arm main bushing, due to lateral free-play in the horizontal plane caused by wear and tear.

Additionally, there is an accelerometer as part of the standard aircraft's navigation system. This is part of an inertial measurement unit, physically located very close to the aircraft's centre of gravity. This existing sensor is used to provide a signal as part of the VHM system, as is discussed in Section 6.

\section{Suspension displacement sensors}

Real-time landing gear suspension travel measurement is often achieved by the use of either a Linear or Rotary Variable Displacement Transformer (LVDT or RVDT), or by the use of simple potentiometers to measure the position of the moving elements depending upon the application. The main drivers are the amount of travel and the desired bandwidth response. In the case of the Demon's system, magnetic proximity sensors were used. They work in saturated mode given the limited travel and hence, it was possible to obtain a high-accuracy and high-bandwidth angular sensing. 
There are three shock absorber pressure sensors on the aircraft in total. These continuously monitor the pressure in each of the three shock absorbers (one per landing gear leg). They are of needle type (see Figure 10), and since the landing gear suffers from shock impact loads, particularly on landing, this type of sensor is very much suited to this application.

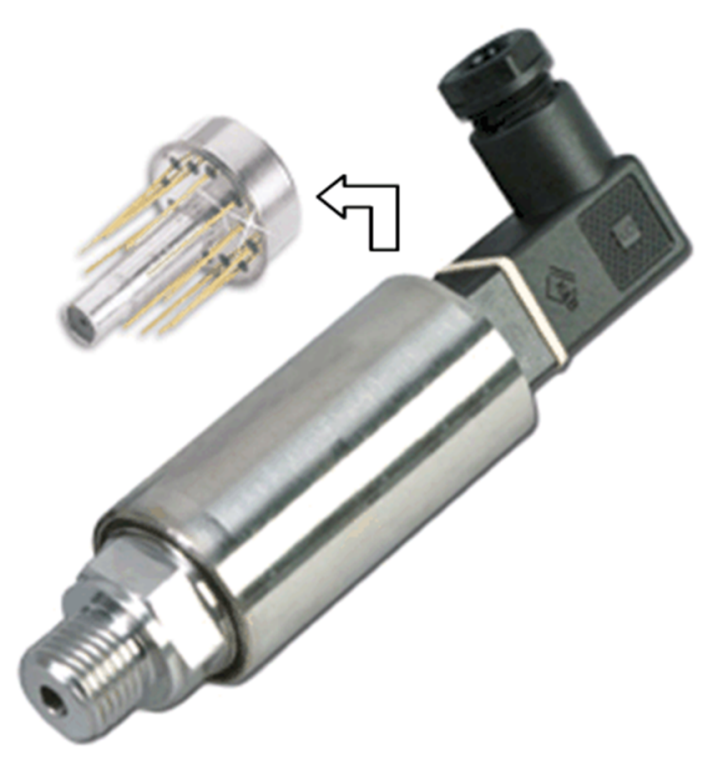

Figure 10. Demon landing gear shock absorber pressure sensor.

The pressure is read from the pressure control valve on the shock absorber. The sensors are mounted inside the fuselage, and so pneumatic tubing connects the sensor to the valve. The installation of the nose landing gear sensor is illustrated in Figure 11. 

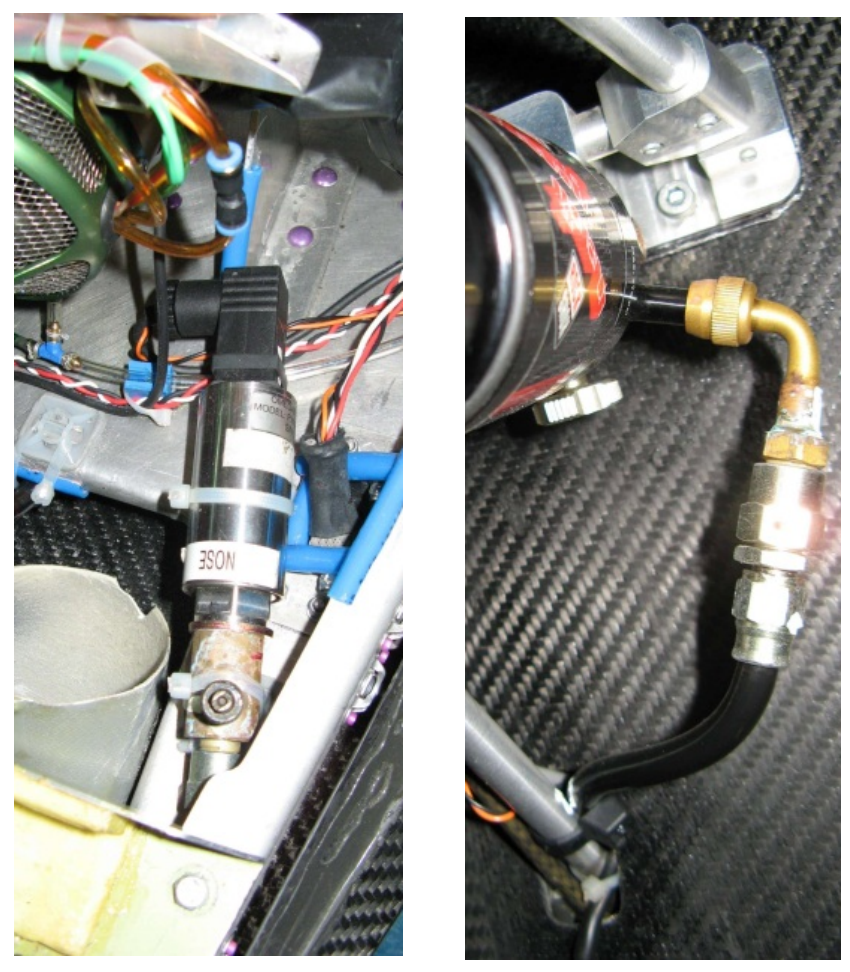

Figure 11. Demon nose landing gear pressure sensor installation and connection to shock absorber.

\section{Flight testing of the Demon VHM system}

In September 2010 the Demon prototype UAV went through its second flight test campaign. This presented the opportunity to flight test the VHM system that had been designed specifically to be retrofitted on the vehicle.

The testing of the VHM system started from some initial ground tests which gave proof of the integrity of the system from both a mechanical and electrical point of view. This was followed by a series of specific tests that took place during the cruise phase of the mission, as well as during take-off and landing. Three examples will be described in the following sections of the document:

- How to detect the correct functionality of a directly monitored sub-system.

- How to detect the correct functionality of an indirectly monitored sub-system. 
- An example of a real, detected failure of an indirectly monitored component that took place during fast taxi trials.

Battery voltage and current monitoring results

The electric quantities (voltage and current) related to the port batteries have been chosen to give an example of how to detect the correct functionality of a directly monitored sub-system. The batteries in question are two custom-made 7.4 V Li-Po batteries, of capacity $6.3 \mathrm{Ah}$ each. From Figure 12 it can be seen that over the 23-minute time history, the voltage of each battery decreases by approximately $\Delta y=$ $0.05 \mathrm{~V}$, mostly happening between $\mathrm{x}=10$ minutes and $\mathrm{x}=15$ minutes. In fact, over said portion of the time history, the current being drained from each battery is at its integral maximum (average $\mathrm{y}=1.3 \mathrm{~A}$ over 5 minutes). This corresponds to a sinusoidal $\pm 100 \%$ command to the conventional ailerons, with approximate period $t=24$ seconds at zero airspeed. The other visible peaks in the time history (in excess of $y=2.5$ A but only for 2 to 8 seconds each) correspond to isolated doublets of the conventional elevators, which involved four actuators instead of only two as in the case of ailerons. The consistency of the voltage trends with respect to the current trends, and the harmonic response of both, shows the correct functionality of the directly monitored sub-system. 


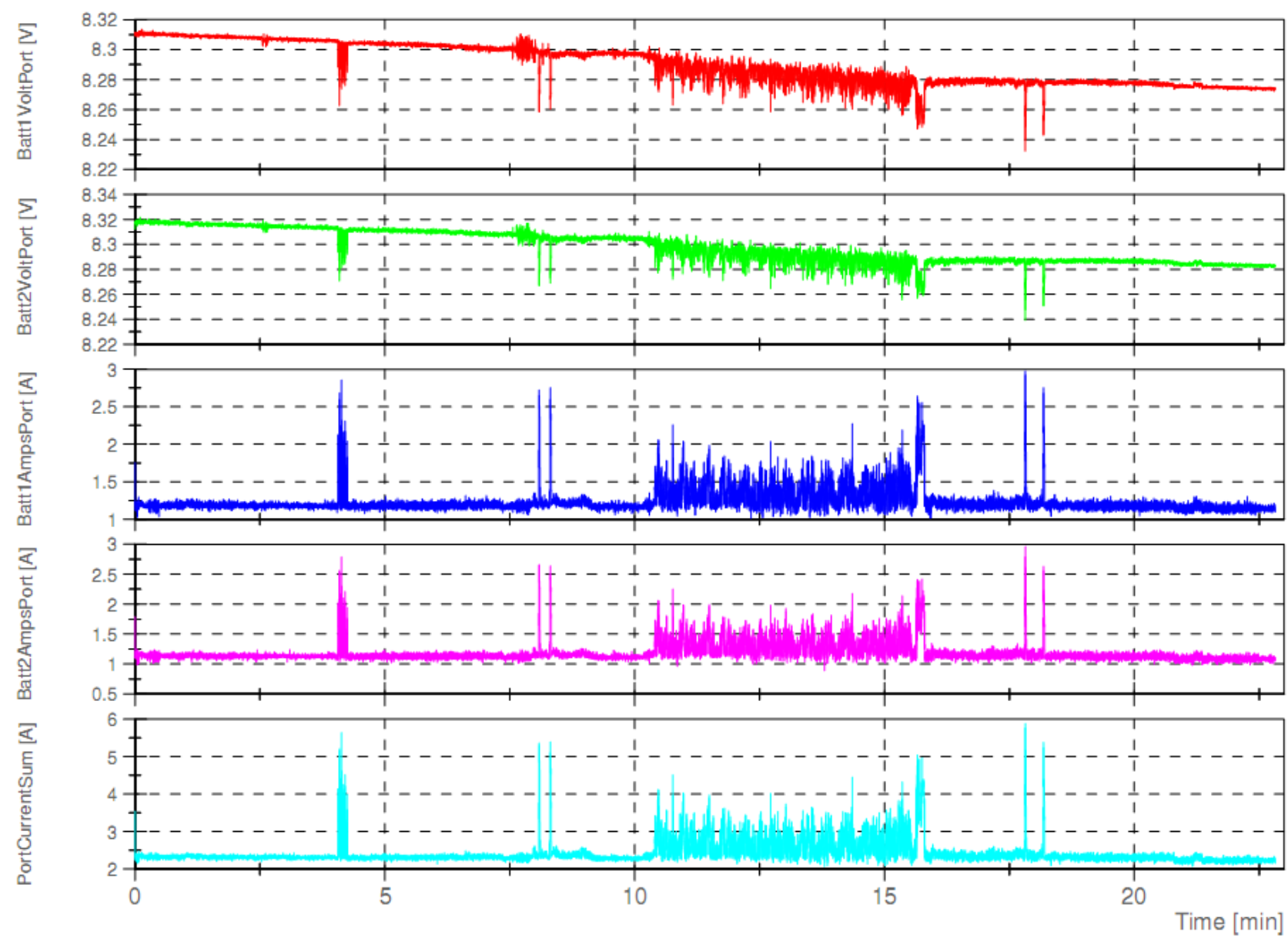

Figure 12. The port batteries data sets.

\section{Wheel braking monitoring results}

A meaningful example of a correctly functioning monitored system can be given by considering the brake-by-wire landing gear sub-system on-board the Demon prototype UAV. In fact, said sub-system is not directly monitored but, the VHM system can indirectly detect the correct functionality of the braking system. This can be done by comparing the respective trends of the main landing gear suspension travel and the internal pressure of the oleo-pneumatic shock absorbers, with reference to the command signal of the brakes. Due to the trailing link design of the main landing gear legs (see Figure 5), the braking torque tends to make the swinging arm rotate upwards, hence compressing the suspension. The force $F$ exerted orthogonally on each end of the swinging arm, which compresses the suspension, can be expressed as a function of the braking torque as follows:

$$
\mathrm{F} \cdot \mathrm{a}=\mathrm{T} \cdot \mathrm{r}
$$


where $a$ is the length of the swinging arm, $T$ is the force of static friction and $r$ is the wheel radius. Therefore:

$$
\mathrm{F}=(\mathrm{N} \cdot \mu \cdot \mathrm{r}) / \mathrm{a}
$$

since $T=N \cdot \mu$, where $N$ is the normal force of gravity and $\mu$ is the static friction coefficient. In Figure 13, the highlighted segment of the time history shows a series of short acceleration runs followed by full braking on a dry tarmac runway. In similar conditions the average braking torque that produces the visible spikes in terms of compression travel and internal pressure is approximately 35 $\mathrm{Nm}$. Once these parameters are know, if the braking command is received and executed by the actuators and no compression travel or pressure increase are observed, then this means that either a loss of wheel/runway grip is being experienced or a loss of pad thickness (wear) is causing the brakes to fail.

The data points are used to capture the faults which are being propagated from the source to destination components, hence can be monitored to know some of the failure modes or aspects of the components. The data transfer points were carefully examined and identified for VHM purposes considering the reliability of the system.

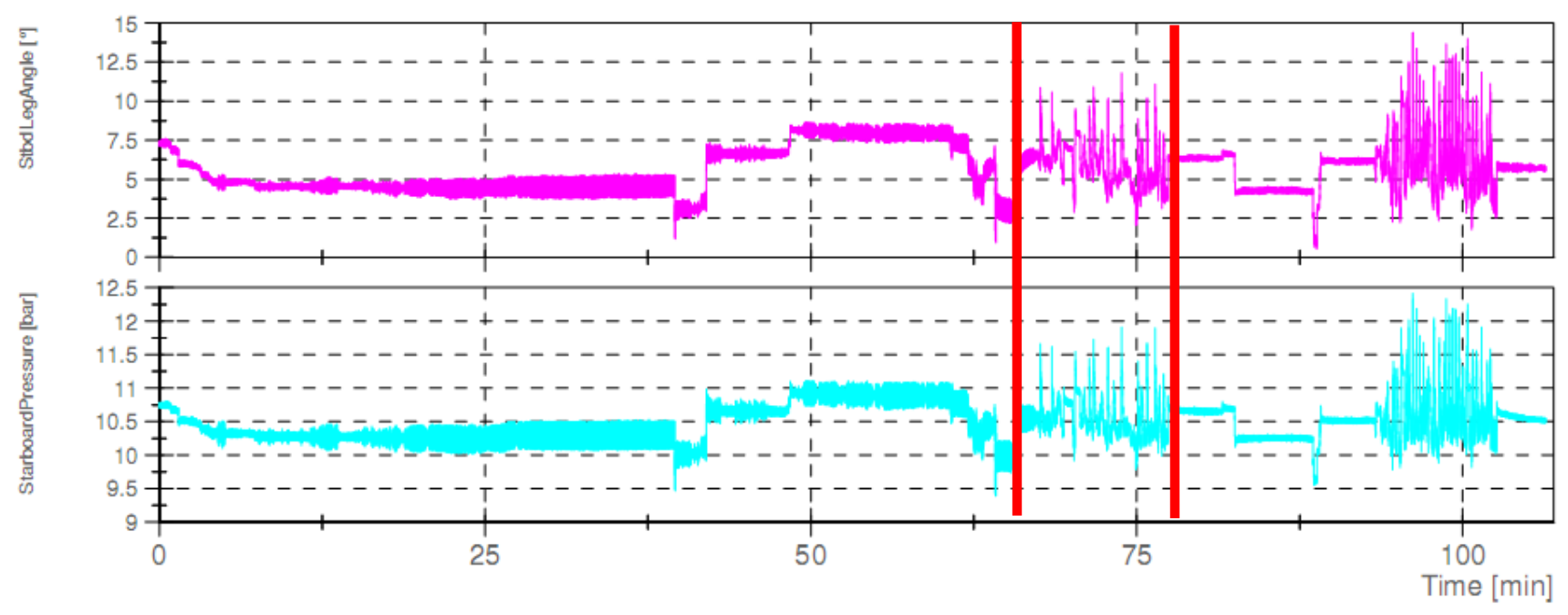

Figure 13. Main landing gear (starboard leg) suspension data sets.

\section{Uneven Tyre Wear Fault Detection}


As an example of a genuine, detected failure of an indirectly monitored component that happened during the flight trials, the most evident instance of such a case is related to the main landing gear legs. Due to a manufacturing imperfection of a disc brake rotor, one of the main wheels had the tendency to lock in a certain position while braking. The skidding which resulted from this caused the semipneumatic tyre to wear abnormally quickly in one precise point of its circumference.

A sequence of five landings was simulated via kinetic energy calculations and an actual series of accelerate-and-stops on a dry tarmac runway. After this exercise, the abrasive effect of the tarmac surface combined with the faulty disc rotor produced a noticeable flat are on one of the main wheels.

During the fast taxi trials this failure was detected retrospectively, by visual inspection. However, by analysing the landing gear health monitoring data, such as that shown in Figure 14, a once-perrevolution failure can be deduced.

Figure 14 shows four traces from the starboard leg sensors; from top to bottom, the wheel hub acceleration in the $\mathrm{x}$ and $\mathrm{z}$ directions, the angle of the leg's trailing arm and, the pressure in the shock absorber. The first 15 minutes of the data represents some normal taxiing of the vehicle, including the acceleration-and stop manoeuvres to test the ground handling and braking. After the braking manoeuvre at 16 minutes, the flat spot on the tyre is caused by the brake locking the wheel and skidding. The data between 16 and 18 minutes shows the aircraft taxiing with the damaged tyre, and the $2 \mathrm{~g}$ vibration generated is apparent from the accelerometer traces. The vehicle then comes to a halt around 18 minutes, and the traces between 18 and 22 minutes show the system noise.

Locally, the radius was reduced by $7 \mathrm{~mm}$ due to a flat face with a chord which was found to be $80 \mathrm{~mm}$ long. This, in turn, while rolling on the runway at up to $2500 \mathrm{rpm}$ during fast taxi generated a cyclic impact load of approximately $2 \mathrm{~g}$ in magnitude. Clearly, the frequency of this vibration should correspond to the rotational speed of the wheel. In order to investigate this the frequency content of the accelerometer signals was analysed.

The wheel speed can be established from the know circumference, and the velocity can be read form the aircraft's navigation system. Further, the $2 \mathrm{~g}$ cyclic impact load frequency can be established by using a spectral analysis of the time variant vibration data in Figure 14. By analysing a five-second period of data around the 17 minute mark (refer to Figure 14) in the frequency domain, we observe a 
vibration frequency of approximately $36 \mathrm{~Hz}$ (see Figure 15). Figure 15 shows the output of a Fourier transform of the time dependant accelerometer data in Figure 14. From the aircraft's navigation system, we establish that the average velocity during that period gives a rotational speed of $2150 \mathrm{rpm}$ $(35.8 \mathrm{~Hz})$. Thus, we can deduce a once-per-revolution fault with the starboard main landing gear.

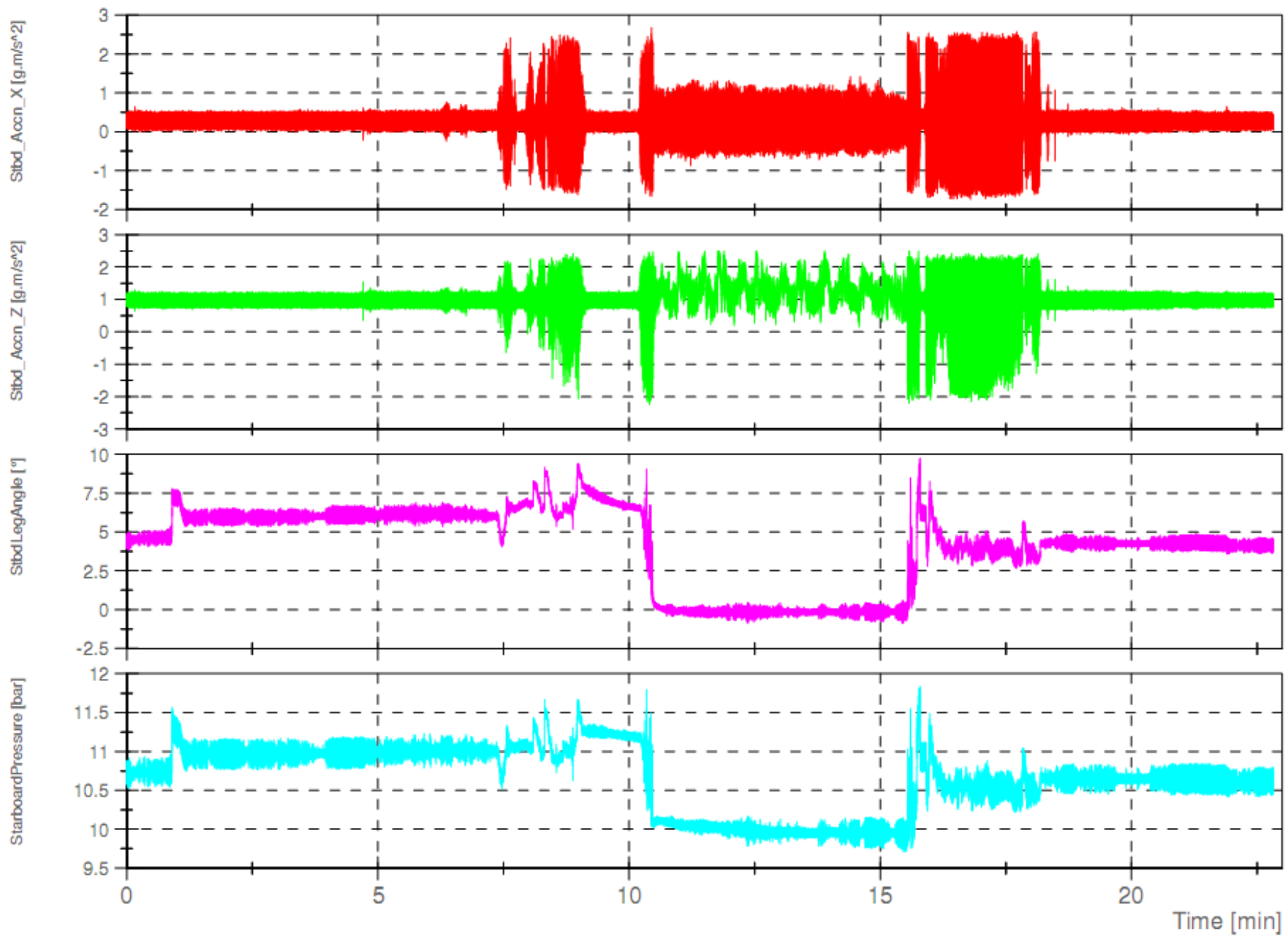

Figure 14. Main landing gear (starboard leg) acceleration data sets. 


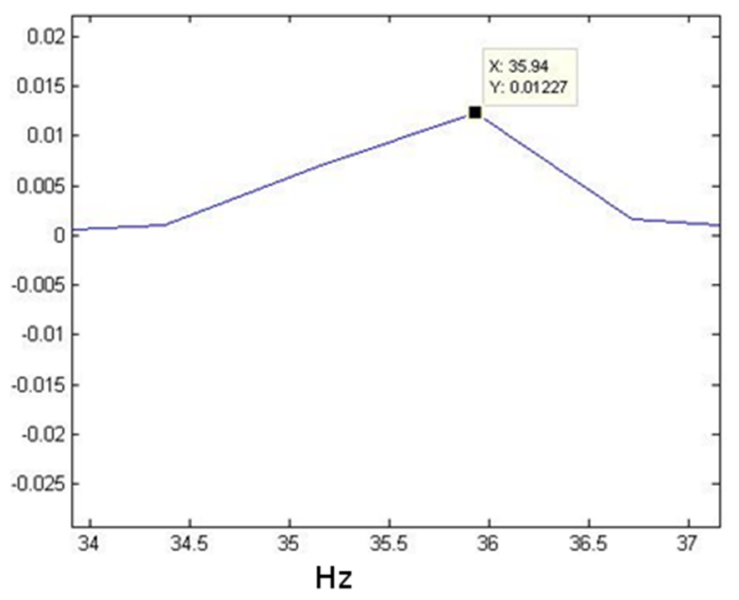

Figure 15. Main landing gear (starboard leg) frequency domain accelerometer vibration data.

\section{Conclusions}

The work reported in this paper provides new insights into the potential for developing health monitoring systems for UAVs. This has been done by designing, integrating and flight testing systems to monitor electrical power system and landing gear health on a pre-existing prototype UAV.

Systems were designed specifically to monitor the UAVs main batteries status, and the landing gear for shimmy due to bearing and damper wear. The battery system was chosen as it is critical to flight safety, as it powers the primary flight control actuators. Landing gear shimmy is a known issue on large aircraft that leads to burdensome maintenance, hence the desire to develop a monitoring system for flight test on the Demon sub-scale demonstrator.

Sensors systems were developed to achieve the desired monitoring outcomes above. This included solving the vital integration issues associated with any flying system, by taking the system fully to the flight testing phase. The health monitoring systems were designed largely using COTS sensor components for rapid and low cost development.

Trials have proven the health monitoring systems attained to be a success. An example of how the status of the main battery system was recorded and analysed has been illustrated. Also, an example of the landing gear suspension monitoring has also been presented. 
Additionally, a case has been presented where a real failure associated with the landing gear (tyre flat spot) has been detected by post-processing data from the sensor systems present to monitor landing gear shimmy, together with data from the aircraft's navigation system.

The work presented in this paper is limited to monitoring the health of the landing gear and main batteries. Further useful work could involve a formal failure mode analysis at the vehicle level. This would enable the design of a truly integrated vehicle health monitoring system to be achieved. Such a system could use a minimised set of sensors sharing data across systems to detect many failure modes associated with the vehicle. Additional further work could usefully involve an in-depth literature review of landing gear health monitoring systems in particular.

\section{Acknowledgements}

The work was supported by the UK Engineering and Physical Sciences Research Council, BAE Systems and the Cranfield University IVHM Centre.

\section{References}

[1] I.K. Jennions, "Integrated Vehicle Health Management: Perspectives on an Emerging Field”, SAE International. 2011.

[2] A.N. Srivastava, "Integrated Vehicle Health Management: Automated Detection, Diagnosis, Prognosis to Enable Mitigation of Adverse Events During Flight. Unpublished NASA Technical Plan. 2009. Accessed $26^{\text {th }}$ July 2012 at http://www.aeronautics.nasa.gov/nra_pdf/ivhm_tech_plan_c1.pdf [3] C.P. Lawson, P.C. Ivey, “Turbomachinery Blade Vibration Amplitude Measurement Through Tip Timing With Capacitance Tip Clearance Probes" Sensors and Actuators A: Physical. Vol. 118, Issue 1, pp. 14-24. January 2005.

[4] “Landing Gear Structural Health Monitoring” SAE International. Standard AIR6168, 2012.

[5] S.E. Woodard, , N.C. Coffey, G.A. Gonzalez, B.D. Taylor, R.R. Brett, K.L. Woodman, B.W.

Weathered, C.H. Rollins, "Development and Flight Testing of an Autonomous Landing Gear HealthMonitoring System”. Unpublished NASA report. Accessed 26 ${ }^{\text {th }}$ July 2012 at http://ntrs.nasa.gov/archive/nasa/casi.ntrs.nasa.gov/20030064479_2003074801.pdf

[5] L. Bagnall, J. Morris, "Landing Gear Health Monitoring”, Aircraft Health Management for New Operational and Enterprise Solutions, IET Seminar on 25-26 June 2008, London. ISBN: 978-0-86341936-2 
[6] A.D. Rosello, “A Vehicle Health Monitoring System for the Space Shuttle Reaction Control System During Re-entry”. Massachusetts Institute of Technology MSc Thesis, 1995. Accessed $26^{\text {th }}$ July 2012 at http://dspace.mit.edu/bitstream/handle/1721.1/46443/33945599.pdf

[7] I.M. Tumer, A. Bajwa, "A Survey of Aircraft Engine Health Monitoring Systems”. AIAA1999-2528. AIAA/ASME/SAE/ASEE Joint Propulsion Conference and Exhibit, 35th, Los Angeles, CA, June 20-24, 1999.

[8] J. Dunston, M. Harrington, "The Application of Open System Architecture for Condition Based Maintenance to Complete IVHM" IEEE Aerospace Conference, 2008.

[9] E.R. Brown, E.E. Moore, N.N. McCollom, A. Hess, "Prognostics and Health Management A Data-Driven Approach to Supporting the F-35 Lightning II" IEEE Aerospace Conference, 2007.

[10] N. Whitaker, "The Application of IVHM to an Aircraft Fuel System" Cranfield University MSc Thesis, 2011.

[11] A. Yarf-Abbasi, A. Clarke, C.P. Lawson, J.P. Fielding, "Design and Development of the Eclipse and Demon Demonstrator UAVs", Paper 316. 26 ${ }^{\text {th }}$ ICAS Congress, Anchorage, Alaska. 14-19 Sept. 2008.

[12] J.P. Fielding, C.P. Lawson, R. Pires, A. Yarf-Abbasi, "Further Development of Eclipse \& Demon Demonstrator UAVs”, CEAS 2009 European Air and Space Conference, Manchester, UK, 26-29 October 2009.

[13] J.P. Fielding, C.P. Lawson, R.M. Pires, G. Monterzino, "Design Build and Flight of the Demon Demonstrator UAV", Paper 2011-6963 $11^{\text {th }}$ AIAA ATIO Conference, Virginia Beach, Virginia, USA. 20-22 Sept. 2011.

[14] J.P. Fielding, C.P. Lawson, R.M. Pires, "Development of the Demon Technology Demonstrator UAV”, Paper 271 27 $7^{\text {th }}$ ICAS Congress, Nice, France. 19-24 Sept. 2010.

[15] G.A.I. Monterzino, C.P. Lawson, “A Methodology For Low-Cost, Rapid-Implementation Solutions For Airframe Systems Of UAV Prototypes", $10^{\text {th }}$ AIAA ATIO Conference, Fort Worth, Texas. 13-15 Sept. 2010

[16] G.A.I. Monterzino, C.P. Lawson, "Development of a Pneumatic System to Enable Flight Without Conventional Control Surfaces", Paper 444. $27^{\text {th }}$ ICAS Congress, Nice, France. 19-24 Sept. 2010. 
2013-05-08

\section{Design manufacturing integration and flight testing of a health monitoring system for a prototype unmanned airborne vehicle}

Lawson, Craig

SAGE

Lawson CP \& Monterzino GA (2014) Design manufacturing integration and flight testing of a health monitoring system for a prototype unmanned airborne vehicle, Proceedings of the Institution of Mechanical Engineers, Part G: Journal of Aerospace Engineering, Volume 228, Issue 7, pp. 997-1009.

htps://doi.org/10.1177/0954410013486856

Downloaded from Cranfield Library Services E-Repository 